PROCEEDINGS OF THE

AMERICAN MATHEMATICAL SOCIETY

Volume 130, Number 7, Pages 2155-2163

S 0002-9939(01)06314-6

Article electronically published on December 31, 2001

\title{
GENUS ONE KNOTS WHICH ADMIT $(1,1)$-DECOMPOSITIONS
}

\author{
HIROSHI MATSUDA \\ (Communicated by Ronald A. Fintushel)
}

\begin{abstract}
We determine the knot types of genus one knots which admit genus one, one bridge decompositions.
\end{abstract}

\section{INTRODUCTION}

A properly embedded arc $t$ in a solid torus $V$ is trivial if there is an embedded disc $C$ in $V$ such that $t \subset \partial C$ and $C \cap \partial V=c \ell(\partial C-t)$. This disc $C$ is called a cancelling disc of $t$. Let $M$ be the 3 -sphere $S^{3}$ or a lens space (not homeomorphic to $S^{1} \times S^{2}$ ). A torus $H$ embedded in $M$ is a genus one Heegaard splitting surface of $M$ if $H$ splits $M$ into two solid tori $V_{1}$ and $V_{2}$. A knot $K$ in $M$ is said to be in genus one 1-bridge position with respect to $H$ if $K$ intersects $H$ transversely in two points and $K \cap V_{i}$ is a trivial arc in $V_{i}$ for $i=1$ and 2. We also say that $K$ is a $(1,1)$-knot, and that $K$ admits a $(1,1)$-decomposition. See [3] for a general definition.

A knot $K$ in $S^{3}$ is satellite if the exterior $E(K)=S^{3}-$ int $N(K)$ of $K$ in $S^{3}$ contains an essential (that is, incompressible and not boundary parallel) torus. A non-trivial knot $K$ in $S^{3}$ is tunnel number one if there exists an arc $\tau$ embedded in $S^{3}$ such that $\tau \cap K=\partial \tau$ and $S^{3}-$ int $N(K \cup \tau)$ is a genus two handlebody. This $\operatorname{arc} \tau$ is called an unknotting tunnel for $K$. It is known that $(1,1)$-knots in $S^{3}$ are tunnel number one. Morimoto, Sakuma and Yokota [8] showed that there are tunnel number one knots which do not admit $(1,1)$-decompositions.

Morimoto and Sakuma 7] determined the knot types of satellite tunnel number one knots in $S^{3}$. These knots are constructed as follows. Let $K_{0}$ be a torus knot of type $(p, q)$ in $S^{3}$ with $p \neq 1$ and $q \neq 1$, and let $L=K_{1} \cup K_{2}$ be a 2-bridge link of type $(\alpha, \beta)$ in $S^{3}$ with $\alpha \geq 4$. Note that $K_{0}$ is a non-trivial knot, and $L$ is neither a trivial link nor a Hopf link. Since $K_{2}$ is the trivial knot in $S^{3}$, there is an orientation preserving homeomorphism $f: E\left(K_{2}\right) \rightarrow N\left(K_{0}\right)$ which takes a meridian $m_{2} \subset \partial E\left(K_{2}\right)$ of $K_{2}$ to a fiber $h \subset \partial N\left(K_{0}\right)=\partial E\left(K_{0}\right)$ of the unique Seifert fibration of $E\left(K_{0}\right)$. The knot $f\left(K_{1}\right) \subset N\left(K_{0}\right) \subset S^{3}$ is denoted by the symbol $K(\alpha, \beta ; p, q)$. Every satellite knot of tunnel number one has the form $K(\alpha, \beta ; p, q)$ for some integers $\alpha, \beta, p$ and $q$. Eudave-Muñoz [4] obtained another description of these knots. These knots are known to admit (1,1)-decompositions.

A non-trivial knot $K$ in $S^{3}$ is free genus one if $K$ bounds a genus one Seifert surface $F$ such that $S^{3}-\operatorname{int} N(F)$ is a genus two handlebody. Goda and Teragaito [5] showed that the set of satellite knots in $S^{3}$ of genus one and tunnel number one is

Received by the editors April 24, 2000 and, in revised form, February 1, 2001.

1991 Mathematics Subject Classification. Primary 57M25.

Key words and phrases. (1,1)-decomposition, genus one Seifert surface. 
the same as the set of $K(8 m, 4 m+1 ; p, q)$, where $m \neq 0$. These knots are known to be free genus one. Ozawa [10] showed that the set of satellite knots in $S^{3}$ of free genus one is also the same as the set of $K(8 m, 4 m+1 ; p, q)$, where $m \neq 0$. In this paper, we prove the following theorem.

Theorem 1.1. Let $K$ be a genus one knot in $S^{3}$ which admits a $(1,1)$-decomposition. Then $K$ is either a 2 -bridge knot $K(4 \alpha \beta-1,2 \alpha)$ or a tunnel number one knot $K(8 m, 4 m+1 ; p, q)$, where $\alpha, \beta, p, q$ and $m$ are non-zero integers, $p \neq 1$ and $q \neq 1$.

Goda and Teragaito raised the following conjecture in [5].

Conjecture 1.2. Non-satellite genus one, tunnel number one knots in $S^{3}$ are 2bridge.

By Theorem 1.1, we obtain the following corollary.

Corollary 1.3. (1,1)-knots in $S^{3}$ satisfy Conjecture 1.2 .

Theorem 1.1 implies that Conjecture 1.2 is equivalent to the following conjecture.

Conjecture 1.4. Genus one, tunnel number one knots in $S^{3}$ admit $(1,1)$-decompositions.

\section{Preliminary theorems}

In this section, we review some results of Hayashi $[6$ which we use in $\S 3$.

A $(1,1)$-decomposition $(M, K)=\left(V_{1}, t_{1}\right) \cup_{H}\left(V_{2}, t_{2}\right)$ of $K$ in $M$ is said to be $K$-reducible if there are a meridian disc $D_{i}$ of $V_{i}$ and a cancelling disc $C_{j}$ of $t_{j}$ in $V_{j}$ such that $D_{i} \cap t_{i}=\emptyset$ and $\partial D_{i} \cap \partial C_{j}=\emptyset$ on $H$ for $(i, j)=(1,2)$ or $(2,1)$. A knot $K$ in $M$ is trivial if $K$ bounds an embedded disc in $M$. The following theorem is Theorem B in [6].

Theorem 2.1. Let $(M, K)=\left(V_{1}, t_{1}\right) \cup\left(V_{2}, t_{2}\right)$ be a $(1,1)$-decomposition of a knot $K$ in $M$. Then $K$ is trivial if and only if the decomposition is $K$-reducible.

A knot $K$ in $M$ is splittable if $M$ contains a 2-sphere $S$ which decomposes $M$ into a 3-ball containing $K$ in its interior and a punctured lens space. Note that every knot in $S^{3}$ is not splittable. Let $F_{1}$ and $F_{2}$ be surfaces properly embedded in $M-$ int $N(K)$. The surfaces $F_{1}$ and $F_{2}$ are said to be roughly isotopic if either (1) they are isotopic in $M-$ int $N(K)$, or (2) the complement of $N(K) \cup F_{1} \cup F_{2}$ contains a once-punctured lens space $X$, and $F_{1}$ and $F_{2}$ are isotopic after $X$ is replaced by a 3-ball. If $K$ is not splittable in $M$, then roughly isotopic surfaces are isotopic in $M-\operatorname{int} N(K)$.

Let $F_{0}$ be an orientable, incompressible and $\partial$-incompressible surface embedded in $M$-int $N(K)$ such that $\partial F_{0}$ consists of $p$ non-meridional loops on $\partial N(K)$. Every component of $\partial F_{0}$ winds around $K$ longitudinally $q$ times for some positive integer $q$. For ease of description, we extend $F_{0}$ naturally in $M$ to obtain a surface $F$ such that $\partial F \subset K$, int $F$ does not have self-intersections, and every point of $K$ is a $p q$ multiple point of $F$. The following theorem is Theorem $\mathrm{A}^{\prime}$ in [6].

Theorem 2.2. Let $(M, K)=\left(V_{1}, t_{1}\right) \cup\left(V_{2}, t_{2}\right)$ be a $(1,1)$-decomposition of a knot $K$ in $M$, and $F$ be a surface as above. Then one of the following holds:

(1) The decomposition is $K$-reducible.

(2) The surface $F$ can be roughly isotoped so that $F \cap V_{1}$ consists of $p q$ cancelling discs of $t_{1}$. 


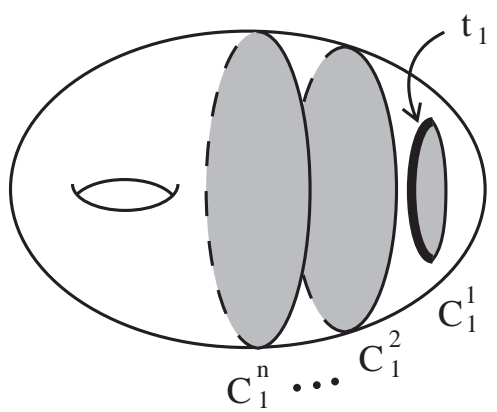

FigURE 1.

(3) We can roughly isotope $F$ so that $F \cap V_{1}$ consists of two kinds of surfaces: pq cancelling discs of $t_{1}$ which are mutually isotopic fixing $t_{1}$ in $V_{1}$, and a non-empty set of parallel peripheral discs, each of which cuts off a 3-ball containing $t_{1}$.

\section{Proof of Theorem 1.1}

Let $K$ be a genus one knot in $S^{3}$ which admits a $(1,1)$-decomposition $\left(S^{3}, K\right)=$ $\left(V_{1}, t_{1}\right) \cup_{H}\left(V_{2}, t_{2}\right)$. Let $F$ denote a genus one Seifert surface of $K$. Note that $K$ is not the trivial knot, and $F \cap E(K)$ is incompressible and $\partial$-incompressible in $E(K)$. The following theorem follows from Theorems 2.1 and 2.2.

Theorem 3.1. Let $K$ and $F$ be as above. Then $F$ can be isotoped so that $F \cap V_{1}$ consists of:

(1) one cancelling disc $C_{1}^{1}$ of $t_{1}$, and

(2) $n-1$ parallel peripheral discs $C_{1}^{2}, \cdots, C_{1}^{n}$, each of which cuts off a 3-ball containing $t_{1}$ in $V_{1}$ as illustrated in Figure 1.

Note that if $n=1$, then condition (2) of Theorem 3.1 is vacuous. We say that $F$ is in standard position if $F$ satisfies the conditions of Theorem 3.1.

Theorem 3.2. Let $K$ be a non-torus genus one knot in $S^{3}$. Suppose $K$ admits a $(1,1)$-decomposition $\left(S^{3}, K\right)=\left(V_{1}, t_{1}\right) \cup_{H}\left(V_{2}, t_{2}\right)$. Then $K$ is either a 2 -bridge knot $K(4 \alpha \beta-1,2 \alpha)$ or a tunnel number one knot $K(8 m, 4 m+1 ; p, q)$, where $\alpha, \beta, p, q$ and $m$ are non-zero integers, $p \neq 1$ and $q \neq 1$.

Since the genus of a torus knot of type $(p, q)$ is $\frac{(|p|-1)(|q|-1)}{2}$ (see, for example, Theorem 7.5.2 in [9]), the genus one torus knots are the right-handed and lefthanded trefoil knots. These trefoil knots are represented as the 2-bridge knots $K(3,2)$ and $K(3,-2)$. Since the trefoil knots are fibered knots, the number of genus one Seifert surfaces of the right-handed and left-handed trefoil knots is one, up to isotopy. See, for example, 11, p. 241] or [11, p. 373]. Then Theorem 1.1 follows from Theorem 3.2.

Proof. Let $F$ be a genus one Seifert surface of $K$. Suppose $F$ is in standard position, that is, $F \cap V_{1}$ consists of one cancelling disc $C_{1}^{1}$ of the arc $t_{1}$ and $n-1$ parallel peripheral $\operatorname{discs} C_{1}^{2}, \cdots, C_{1}^{n}$. If $n=1$, then $F \cap V_{1}$ consists only of one cancelling $\operatorname{disc} C_{1}^{1}$ of $t_{1}$. We assume that the 3 -ball cut off by $C_{1}^{k}(k=2,3, \cdots, n)$ in $V_{1}$ contains $C_{1}^{1}$ and $C_{1}^{j}$ for $j=k-1$. See Figure 1 . Let $C_{2}$ be a cancelling disc of $t_{2}$ 
in $V_{2}$. If (int $\left.\left(C_{1}^{1} \cap H\right)\right) \cap\left(\operatorname{int}\left(C_{2} \cap H\right)\right)=\emptyset$, then $K$ can be isotoped onto $H$, and $K$ is a torus knot. Hence we may assume $\left(\operatorname{int}\left(C_{1}^{1} \cap H\right)\right) \cap\left(\operatorname{int}\left(C_{2} \cap H\right)\right) \neq \emptyset$.

Isotope $C_{2}$ so that $N\left(t_{2} ; C_{2}\right) \cap F=\emptyset$, where $N\left(t_{2} ; C_{2}\right)$ denotes a neighborhood of $t_{2}$ in $C_{2}$. The complexity $c(F)$ for a cancelling disc $C_{2}$ of $t_{2}$ and a Seifert surface $F$ in standard position is defined as the pair $\left(n,\left|C_{2} \cap F\right|\right)$. We assign the standard lexicographic ordering to this complexity function $c(F)$. We suppose that $c(F)$ is minimal among all cancelling discs of $t_{2}$ and all genus one Seifert surfaces of $K$ which are isotopic to $F$. We may assume, by the minimality of $c(F)$ and the incompressibility of $F \cap E(K)$ in $E(K)$, that each component $s$ of $C_{2} \cap F$ in $C_{2}$ is a properly embedded arc such that both endpoints of $s$ lie on $C_{2} \cap H$. Since $\left(\operatorname{int}\left(C_{1}^{1} \cap H\right)\right) \cap\left(\operatorname{int}\left(C_{2} \cap H\right)\right) \neq \emptyset,\left|C_{2} \cap F\right| \neq 0$.

Let $\delta$ be an outermost disc separated by $C_{2} \cap F$ in $C_{2}$ such that $\partial \delta$ is disjoint from $t_{2}$. Let $\gamma$ denote the $\operatorname{arc} \delta \cap H$. An isotopy of $F$ along $\delta$ produces a band in $V_{1}$ which connects components of $F \cap V_{1}$. The core of the band is $\gamma$. Let $F^{(1)}$ denote the image of $F$ after this isotopy.

First suppose $\gamma$ connects $C_{1}^{1}$ and $C_{1}^{2}$. Then $F^{(1)}$ is in standard position with $n-2$ parallel peripheral discs, and $c\left(F^{(1)}\right)<c(F)$. Next suppose $\gamma$ connects $C_{1}^{i}$ and $C_{1}^{i+1}(i=2,3, \cdots, n-1)$. Then $F^{(1)}$ can be isotoped to $F^{\prime}$ which is in standard position with $n-3$ parallel peripheral discs, and $c\left(F^{\prime}\right)<c(F)$. Next suppose both endpoints of $\gamma$ lie on $C_{1}^{1} \cap H$, and that a subarc of $C_{1}^{1} \cap H$ together with $\gamma$ cobounds a disc on $H$. The disc $C_{1}^{1}$ is deformed to an annulus by an isotopy of $F$ to $F^{(1)}$. Note that $\left|C_{2} \cap F^{(1)}\right|=\left|C_{2} \cap F\right|-1$. There is a compressing disc $\Delta$ in $V_{1}$ for this annulus such that $\Delta$ is disjoint from $C_{1}^{j}$ for $j=2,3, \cdots, n$. Since $F^{(1)} \cap E(K)$ is incompressible in $E(K), \partial \Delta$ bounds a disc $\Delta^{\prime}$ on $F^{(1)}$. The 2-sphere $\Delta \cup \Delta^{\prime}$ bounds a 3-ball whose interior is disjoint from $F^{(1)}$ and $K$. Isotope $F^{(1)}$ to $F^{\prime}$ so that $\Delta^{\prime}$ is isotoped along the 3-ball to $\Delta$. Then $F^{\prime}$ is in standard position with $n-1$ or less parallel peripheral discs, and $\left|C_{2} \cap F^{\prime}\right| \leq\left|C_{2} \cap F^{(1)}\right|$. Therefore $c\left(F^{\prime}\right)<c(F)$. Next suppose both endpoints of $\gamma$ lie on $C_{1}^{i} \cap H(i=2,3, \cdots, n)$, and a subarc of $C_{1}^{i} \cap H$ together with $\gamma$ cobounds a disc on $H$. Similar arguments as above show that we can isotope $F^{(1)}$ to $F^{\prime}$ so that $F^{\prime}$ is in standard position and $c\left(F^{\prime}\right)<c(F)$. Hence both endpoints of $\gamma$ lie on $C_{1}^{n} \cap H$, and a subarc of $C_{1}^{n} \cap H$ together with $\gamma$ does not cobound a disc on $H$, that is, forms a torus knot on $H$.

Let $D_{1}^{n}$ be the component of $F^{(1)} \cap V_{1}$ which contains the image of $C_{1}^{n}$. Note that $D_{1}^{n}$ is an annulus. If $n=1$, then we proceed to the paragraph just before Lemma 3.3. Suppose $n \geq 2$. The annulus $D_{1}^{n}$ is properly embedded in $V_{1}$. Let $d$ be a disc in $V_{1}$ such that $d \cap D_{1}^{n}$ is an essential arc in $D_{1}^{n}, d \cap H$ is an arc on $H$, and that $F$ is obtained by an isotopy of $F^{(1)}$ along $d$. Let $\delta^{(1)}$ be an outermost disc separated by $C_{2} \cap F^{(1)}$ in $C_{2}$ such that $\partial \delta^{(1)}$ is disjoint from $t_{2}$. Let $\gamma^{(1)}$ denote the $\operatorname{arc} \delta^{(1)} \cap H$.

First suppose $\gamma^{(1)}$ connects $C_{1}^{n-1}$ and $D_{1}^{n}$. We may assume, after an isotopy of $d$, that $d$ is disjoint from $\gamma^{(1)}$ on $H$. Isotope $F^{(1)}$ to $F^{\prime}$ along $d$ and $\delta^{(1)}$. Then $F^{\prime}$ can be further isotoped to $F^{\prime \prime}$ which is in standard position with $n-3$ parallel peripheral discs (respectively without parallel peripheral discs) if $n \geq 4$ (resp. if $n=2$ and 3). Therefore $c\left(F^{\prime \prime}\right)<c(F)$. Similar arguments as above show that both endpoints of $\gamma^{(1)}$ lie on either $D_{1}^{n}$ or $C_{1}^{n-1}$. Next suppose both endpoints of $\gamma^{(1)}$ lie on $D_{1}^{n}$. The torus $H$ is separated by $\partial D_{1}^{n}$ into two annuli. We may assume, by similar arguments as above, that $\gamma^{(1)}$ is an essential arc of one of the two annuli on $H$. Let $\widetilde{F^{(1)}}$ be the image of $F^{(1)}$ after an isotopy along $\delta^{(1)}$, and $\widetilde{D_{1}^{n}}$ 
be the component of $\widetilde{F^{(1)}} \cap V_{1}$ which contains the image of $D_{1}^{n}$. Then $\widetilde{D_{1}^{n}}$ is a oncepunctured torus, and there is an annulus on the Seifert surface $\widetilde{F^{(1)}}$ cobounded by $K$ and $\partial \widetilde{D_{1}^{n}}$. This shows that $K$ is isotopic to the circle $\partial \widetilde{D_{1}^{n}}$, and $K$ is the trivial knot. Next suppose that both endpoints of $\gamma^{(1)}$ lie on $C_{1}^{n-1}$. Similar arguments as above show that $\gamma^{(1)}$ and a subarc of $C_{1}^{n-1} \cap H$ form a torus knot on $H$ which is isotopic to a component of $\partial D_{1}^{n}$.

Let $F^{(k)}(k=2,3, \cdots, n)$ denote the image of $F^{(k-1)}$ after an isotopy of $F^{(k-1)}$ along an outermost disc $\delta^{(k-1)}$ of $C_{2} \cap F^{(k-1)}$ in $C_{2}$ which is disjoint from $t_{2}$. Similar arguments as above show that both endpoints of the arc $\gamma^{(k)}=\delta^{(k)} \cap H$ $(k=2,3, \cdots, n-1)$ lie on $C_{1}^{n-k}$, and that the arc $\gamma^{(k)}$ and a subarc of $C_{1}^{n-k} \cap H$ form a torus knot on $H$ which is isotopic to a component of $\partial D_{1}^{n}$. Since $K$ is not a torus knot, $C_{2} \cap F^{(k)} \neq \emptyset$ for $k<n$. The intersection $F^{(k)} \cap V_{1}$ consists of $k$ annuli and $n-k$ discs for $k=1, \cdots, n$. Let $D_{1}^{j}(j=1, \cdots, n-1)$ denote the component of $F^{(n)} \cap V_{1}$ which contains the image of $C_{1}^{j}$.

Suppose $n \geq 1$. Let $G^{1}, \cdots, G^{m}$ be components of $F^{(n)} \cap V_{2}$. Since $F^{(n)} \cap H$ consists of one arc and $2 n-1$ loops, $\chi\left(F^{(n)} \cap H\right)=1$. A calculation of Euler characteristics shows that

$$
-1=\chi\left(F^{(n)}\right)=\left(\chi\left(D_{1}^{1}\right)+\cdots+\chi\left(D_{1}^{n}\right)\right)+\left(\chi\left(G^{1}\right)+\cdots+\chi\left(G^{m}\right)\right)-\chi\left(F^{(n)} \cap H\right),
$$

so $\chi\left(G^{1}\right)+\cdots+\chi\left(G^{m}\right)=0$.

Lemma 3.3. Every component of $F^{(n)} \cap V_{2}$ is an annulus.

Proof. Suppose there is a disc component $G$ of $F^{(n)} \cap V_{2}$. The component $G \cap H$ is either an arc component of $D_{1}^{1} \cap H$ or a loop component of $D_{1}^{j} \cap H(j=1, \cdots, n)$.

First suppose $G \cap H$ is an arc component of $D_{1}^{1} \cap H$. Note that $t_{2}$ is contained in $\partial G$. The construction of $F^{(n)}$ shows that there is a cancelling disc $C_{1}$ of $t_{1}$ with $C_{1} \cap F^{(n)}=t_{1}$. The union of discs $C_{1}$ and $G$ shows that $K=t_{1} \cup t_{2}$ is isotopic to a torus knot on $H$.

Next suppose $G \cap H$ is a loop component of $D_{1}^{j} \cap H$. Suppose $C_{2} \cap G \neq \emptyset$. Each component of $C_{2} \cap G$ is a properly embedded arc in $C_{2}$ and $G$. Let $\gamma_{G}$ denote an outermost arc of $C_{2} \cap G$ in $G$, and $\delta_{G}$ denote the corresponding outermost disc in $G$. Let $\gamma_{C}$ be the arc of $C_{2} \cap G$ in $C_{2}$ which corresponds to $\gamma_{G}$, and $\delta_{C}$ be the disc in $C_{2}$ cobounded by $\gamma_{C}$ and a subarc of $C_{2} \cap H$. Let $C_{2}^{\prime}$ be the disc $\left(C_{2}-\delta_{C}\right) \cup \delta_{G}$ isotoped slightly off $\delta_{G}$. This disc $C_{2}^{\prime}$ is a cancelling disc of $t_{2}$ with $\left|C_{2}^{\prime} \cap G\right|<\left|C_{2} \cap G\right|$. So we may suppose $C_{2} \cap G=\emptyset$. Then the arc $t_{2}$ is a trivial arc in the 3 -ball $c \ell\left(V_{2}-N(G)\right)$. Let $C_{1}$ be a cancelling disc of $t_{1}$ with $C_{1} \cap F^{(n)}=t_{1}$. The arc $t_{1}$ can be isotoped into $H-G$ along $C_{1}$, and $t_{1}$ may be regarded as a trivial arc in the 3-ball $V_{1} \cup N(G)$. Therefore $K=t_{1} \cup t_{2}$ is the trivial knot in $S^{3}=\left(V_{1} \cup N(G)\right) \cup\left(c \ell\left(V_{2}-N(G)\right)\right)$.

Since $\chi\left(G^{1}\right)+\cdots+\chi\left(G^{m}\right)=0$ and no component of $F^{(n)} \cap V_{2}$ is a disc, $\chi\left(G^{1}\right)=$ $\cdots=\chi\left(G^{m}\right)=0$ and every component of $F^{(n)} \cap V_{2}$ is an annulus.

Note that $F^{(n)} \cap H$ consists of one arc and $2 n-1$ loops which are essential in $F^{(n)}$. Since $F^{(n)} \cap E(K)$ is incompressible in $E(K)$, each component of $F^{(n)} \cap V_{1}$ (respectively $F^{(n)} \cap V_{2}$ ) is incompressible in $V_{1}$ (resp. $V_{2}$ ). Let $D_{2}^{1}$ denote the component of $F^{(n)} \cap V_{2}$ such that $t_{2}$ is contained in $\partial D_{2}^{1}$. Let $D_{2}^{2}, \cdots, D_{2}^{n}$ denote the other components of $F^{(n)} \cap V_{2}$. Since $D_{2}^{j}(j=2,3, \cdots, n)$ is an annulus properly embedded in $V_{2}, D_{2}^{j}$ is boundary parallel in $V_{2}$.

First suppose $\left(\right.$ int $\left.C_{2}\right) \cap D_{2}^{1}=\emptyset$. Since $D_{2}^{1}$ is incompressible in $V_{2}$, the union $C_{2} \cup D_{2}^{1}$ is an annulus which is boundary parallel in $V_{2}$. Then we can find a disc 


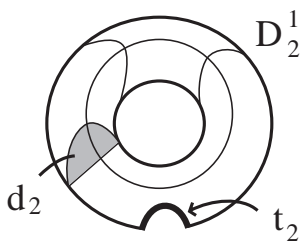

( 1 )

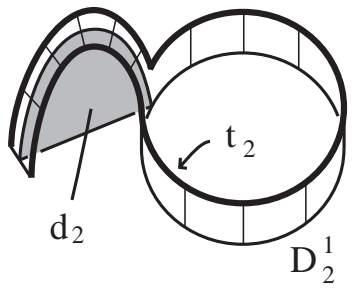

( 2 )

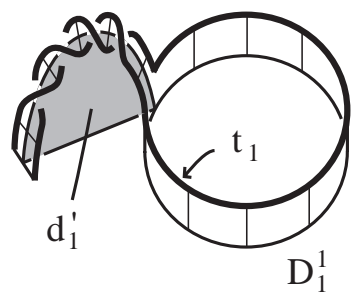

( 3 )

FIGURE 2.

$d_{2}$ in $V_{2}$ such that $d_{2} \cap D_{2}^{1}$ is an essential arc on $D_{2}^{1}, d_{2} \cap H$ is an arc on $H$, and $\left(d_{2} \cap D_{2}^{1}\right) \cup\left(d_{2} \cap H\right)=\partial d_{2}$. See Figure $2(1)$.

Next suppose $\left(\right.$ int $\left.C_{2}\right) \cap D_{2}^{1} \neq \emptyset$. Let $\gamma$ be an outermost arc of $C_{2} \cap D_{2}^{1}$ in $C_{2}$, and $d_{2}$ be the corresponding outermost disc in $C_{2}$ such that $\partial d_{2}$ is disjoint from $t_{2}$. Similar arguments as in the proof of Lemma 3.3 show that $\gamma$ corresponds to an essential arc on $D_{2}^{1}$. Then $d_{2} \cap D_{2}^{1}$ is an essential arc on $D_{2}^{1}, d_{2} \cap H$ is an arc on $H$, and $\left(d_{2} \cap D_{2}^{1}\right) \cup\left(d_{2} \cap H\right)=\partial d_{2}$. See Figure 2 (1).

Suppose $n \geq 2$. If $n=1$, we skip this paragraph. Suppose the solid torus of a parallelism between an annulus $D_{2}^{i}(i=2,3, \cdots, n)$ and an annulus on $H$ does not contain $D_{2}^{1}$. By retaking $D_{2}^{i}$ as an outermost annulus, if necessary, we may assume that the interior of the solid torus contains no component of $F^{(n)} \cap V_{2}$. Suppose $D_{2}^{i}$ connects two boundary components of $D_{1}^{n}$. Then the union $D_{1}^{n} \cup D_{2}^{i}$ is a torus. This contradicts the fact that $F^{(n)}$ is a Seifert surface. Suppose $D_{2}^{i}$ connects loop components of $\partial D_{1}^{j}$ and $\partial D_{1}^{j+1}(j=1, \cdots, n-1)$. Isotope $D_{2}^{i}$ into $V_{1}$ along the solid torus of the parallelism; then $F^{(n)}$ is isotoped to $F^{\prime}$ so that $F^{\prime} \cap H$ consists of one arc and $2 n-3$ loops which are essential in $F^{\prime}$, and that each component of $F^{\prime} \cap V_{1}$ (resp. $F^{\prime} \cap V_{2}$ ) is an annulus in $V_{1}$ (resp. $V_{2}$ ). Therefore $F^{\prime}$ satisfies Lemma 3.3 and $\left|F^{\prime} \cap H\right|=\left|F^{(n)} \cap H\right|-2$. So we may suppose the interior of the solid torus $W$ of a parallelism in $V_{2}$ between an annulus $D_{2}^{i}$ and an annulus on $H$ contains $D_{2}^{1}$ and no $D_{2}^{j}(j=2,3, \cdots, n)$. Assume $d_{2} \cap D_{2}^{i} \neq \emptyset$. We may suppose that each component of $d_{2} \cap D_{2}^{i}$ is an essential arc in $D_{2}^{i}$. Let $\delta$ be an outermost disc separated by $d_{2} \cap D_{2}^{i}$ in $d_{2}$ such that $\partial \delta$ is disjoint from $d_{2} \cap D_{2}^{1}$. Note that each component of $\partial D_{2}^{i}$ is parallel to the loop component of $D_{2}^{1}$ on $H$. Then $\delta$ is a meridian disc of the solid torus $W^{\prime}=c \ell\left(V_{2}-W\right)$. This shows that each component of $\partial D_{2}^{i}$ winds around $\partial V_{2}$ once longitudinally, and the solid torus $W^{\prime}$ realizes a parallelism between $D_{2}^{i}$ and an annulus on $H$ such that $W^{\prime}$ does not contain $D_{2}^{1}$. Hence we may assume $d_{2} \cap D_{2}^{i}=\emptyset$.

Suppose $n \geq 1$. Let $\ell_{2}$ be the loop component of $D_{2}^{1} \cap H$. Isotope $N\left(D_{2}^{1} ; F^{(n)}\right)$ so that the union of $N\left(\ell_{2} ; D_{2}^{1}\right)$ and $N\left(d_{2} \cap D_{2}^{1} ; D_{2}^{1}\right)$ is $D_{2}^{1}$. See Figure 2 (2). The loop components of $F^{(n)} \cap H$ separate $H$ into $2 n-1$ annuli. Let $A$ be the component of these annuli on $H$ such that $A$ contains the arc component of $F^{(n)} \cap H$. Let $c$ be an essential arc in $A$ such that the $\operatorname{arc} d_{2} \cap H$ is contained in $c$. We can take a meridian disc $Q$ of $V_{2}$ so that $d_{2}$ is contained in $Q$. Note that $c$ is contained in $\partial Q$.

The construction of $F^{(n)}$ shows that there is a disc $d_{1}$ in $V_{1}$ such that $d_{1} \cap D_{1}^{j}=\emptyset$ $(j=2,3, \cdots, n), d_{1} \cap D_{1}^{1}$ is an essential arc in $D_{1}^{1}, d_{1} \cap H$ is an arc on $A$, and $\left(d_{1} \cap D_{1}^{1}\right) \cup\left(d_{1} \cap H\right)=\partial d_{1}$. Isotope $d_{1}$ so that the two points $\partial\left(d_{1} \cap H\right)$ are 
contained in $c$, and that there is an essential arc $a$ of $A$ such that $a$ is disjoint from $c$ and $d_{1} \cap H$. Then the arc $d_{1} \cap H$ intersects the arc $d_{2} \cap H$ transversely on $H$. We regard the arc $d_{1} \cap H$ as being very short, and we take a neighborhood $N(c ; A)$ so that it contains the arc $d_{1} \cap H$. Let $\ell_{1}$ be the loop component of $D_{1}^{1} \cap H$. We may regard $D_{1}^{1}$ as the union of $N\left(\ell_{1} ; D_{1}^{1}\right)$ and $N\left(d_{1} \cap D_{1}^{1} ; D_{1}^{1}\right)$. The $\operatorname{arc} d_{1} \cap D_{1}^{1}$ together with a subarc of $c$ cobounds a disc $d_{1}^{\prime}$ in $V_{1}$. See Figure 2 (3). Note that $t_{1}$ may intersect $d_{1}^{\prime}$.

First suppose $d_{1}^{\prime} \cap H=d_{2} \cap H$. Then the union $D_{1}^{1} \cup D_{2}^{1}$ is a Seifert surface of $K$, and $\ell_{1}=\ell_{2}$ on $H$. This Seifert surface is said to be of type I. The disc which is a union of $d_{1}^{\prime}$ and $d_{2}$ shows that the Seifert surface of type I is constructed as a plumbing of the two annuli

$$
N\left(\ell_{1} ; D_{1}^{1}\right) \cup N\left(\ell_{2} ; D_{2}^{1}\right) \quad \text { and } \quad N\left(\partial d_{1}^{\prime} \cap D_{1}^{1} ; D_{1}^{1}\right) \cup N\left(d_{2} \cap D_{2}^{1} ; D_{2}^{1}\right) .
$$

Since the Seifert surface $D_{1}^{1} \cup D_{2}^{1}$ is orientable, a sub-disc of $N\left(d_{1}^{\prime} \cap H ; A\right)$ together with the disc $N\left(\partial d_{1}^{\prime} \cap D_{1}^{1} ; D_{1}^{1}\right)$ forms an annulus $P$ such that $\partial d_{1}^{\prime}$ is a core of $P$. Let $k_{1}$ and $k_{2}$ be the boundary components of $P$, and let $\beta$ denote the linking number of $k_{1}$ with $k_{2}$, where the orientations of $k_{1}$ and $k_{2}$ are induced from an orientation of $P$. If $\beta=0$, then the disc $d_{1}^{\prime} \cup d_{2}$ is a compressing disc of the Seifert surface $D_{1}^{1} \cup D_{2}^{1}$, and $K$ is the trivial knot. First suppose $\ell_{1}$ is a torus knot of type $(1, \alpha)$ on $\partial V_{1}$. The loop $\ell_{1}$ is the trivial knot in $S^{3}$. If $\alpha=0$, that is, $\ell_{1}$ is the boundary of a meridian disc of $V_{1}$, then the meridian disc is a compressing disc of the Seifert surface $D_{1}^{1} \cup D_{2}^{1}$, and $K$ is the trivial knot. The construction of the Seifert surface of type I shows that $K$ is a 2 -bridge knot $K(4 \alpha \beta-1,2 \alpha)$, where $\alpha \neq 0$ and $\beta \neq 0$. Note that every genus one 2-bridge knot is represented as $K(4 \alpha \beta-1,2 \alpha)$ for some non-zero integers $\alpha$ and $\beta$. See, for example, Proposition 12.25 in [2]. Next suppose $\ell_{1}$ is a torus knot of type $(\alpha, 1)$ on $\partial V_{1}$. The same arguments as above show, after interchanging the roles of $V_{1}$ and $V_{2}$, that $K$ is a 2-bridge knot $K(4 \alpha \beta-1,2 \alpha)$. Next suppose $\ell_{1}$ is a torus knot of type $(p, q)$ on $\partial V_{1}$, where $p \neq 1$ and $q \neq 1$. Let $H_{W}$ be a genus one Heegaard splitting surface of $S^{3}$, and $W_{1}, W_{2}$ be the solid tori in $S^{3}$ with $W_{1} \cap W_{2}=\partial W_{1}=\partial W_{2}=H_{W}$. Let $h$ be a loop on $H_{W}$ which is the boundary of a meridian disc of $W_{2}$, and let $w_{2}$ be a loop in $W_{2}$ which is isotopic to a core of $W_{2}$. Let $U$ be the solid torus which is a union of $N\left(D_{1}^{1} \cup D_{2}^{1}\right)$ and $N\left(d_{1}^{\prime} \cup d_{2}\right)$. We assume $\partial U$ intersects $H$ in two loops. Let $u$ be a component of $\partial U \cap H$. Let $f$ be a homeomorphism from $U$ to $W_{1}$ such that $f(u)=h$. The image $f(K)$ is a knot in $W_{1}$. Figure 3 illustrates a pair $\left(W_{1}, f(K)\right)$. This pair is uniquely determined, up to isotopy of $f(K)$ in $W_{1}$. Then the union $f(K) \cup w_{2}$ in $S^{3}=W_{1} \cup W_{2}$ is a 2-bridge link $K(8 \beta, 4 \beta+1)$. This shows that $K$ is a tunnel number one knot $K(8 \beta, 4 \beta+1 ; p, q)$. Since the union of the $\operatorname{arcs} \partial d_{1}^{\prime} \cap D_{1}^{1}, \partial d_{2} \cap D_{2}^{1}$ and the loop $\ell_{1}$ is a deformation retract of $D_{1}^{1} \cup D_{2}^{1}, S^{3}-\operatorname{int} N\left(D_{1}^{1} \cup D_{2}^{1}\right)$ is not a handlebody.

Next suppose the union of the two $\operatorname{arcs} d_{1}^{\prime} \cap H$ and $d_{2} \cap H$ is the arc $c$. Suppose $n \geq 2$. Then $D_{1}^{1}$ connects $D_{2}^{1}$ and $D_{2}^{k}$, and $D_{2}^{1}$ connects $D_{1}^{1}$ and $D_{1}^{j}(j, k \neq 1)$. Isotope $D_{1}^{1}$ (resp. $\left.D_{2}^{1}\right)$ into $V_{2}$ (resp. $V_{1}$ ) so that the $\operatorname{disc} N\left(\partial d_{1}^{\prime} \cap D_{1}^{1} ; D_{1}^{1}\right)$ (resp. $\left.N\left(d_{2} \cap D_{2}^{1} ; D_{2}^{1}\right)\right)$ is isotoped along the disc $d_{1}^{\prime}$ (resp. $d_{2}$ ). The union of $D_{1}^{1}$ and $D_{2}^{k}$ (resp. $D_{2}^{1}$ and $D_{1}^{j}$ ) is isotoped to an annulus in $V_{2}$ (resp. $V_{1}$ ). See Figure 4 for a sketch of this isotopy. Figure 4 illustrates an intersection of the surface $D_{1}^{1} \cup D_{2}^{k} \cup D_{2}^{1} \cup D_{1}^{j}$ with a plane in $S^{3}$ which contains $Q$. Let $F^{\prime}$ denote the image of $F^{(n)}$ after this isotopy. Then $F^{\prime} \cap H$ consists of one arc and $2 n-3$ loops which are essential in $F^{\prime}$, and every component of $F^{\prime} \cap V_{1}$ (resp. $F^{\prime} \cap V_{2}$ ) is an annulus 


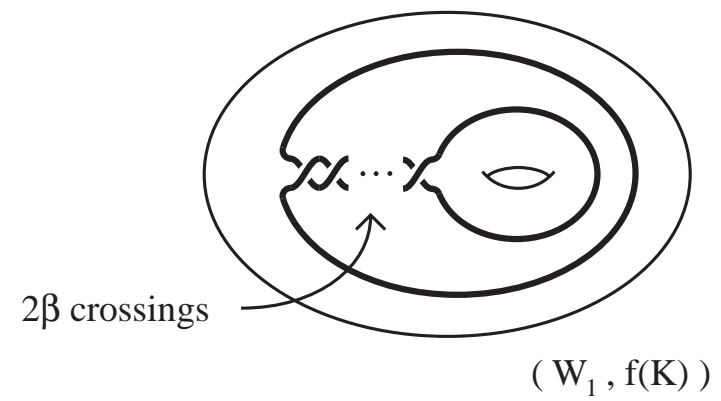

FiguRE 3.
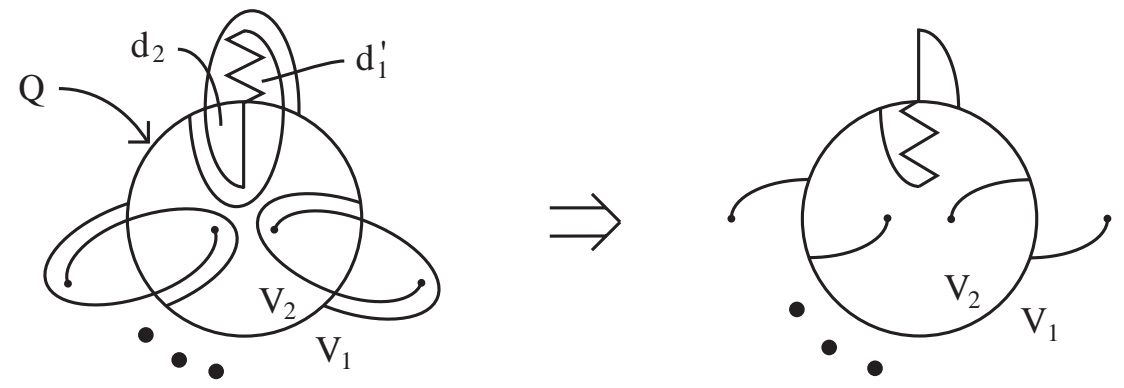

FIGURE 4

in $V_{1}$ (resp. $V_{2}$ ). So we may suppose $n=1$. The union $D_{1}^{1} \cup D_{2}^{1}$ is a Seifert surface of $K$, and $\ell_{1}=\ell_{2}$ on $H$. This Seifert surface is said to be of type II. Let $P$ be the same annulus as in the previous paragraph, and let $\beta$ be the linking number as above. First suppose $\ell_{1}=\ell_{2}$ is a torus knot of type $(\alpha, 1)$ on the torus $\partial V_{2}$. The same arguments as in the previous paragraph show $\alpha \neq 0$. The $\operatorname{arcs} d_{2} \cap D_{2}^{1}$ and $c \ell\left(\partial Q-d_{2} \cap H\right)$ cobound a disc $d_{2}^{\prime}$ in $Q$. The discs $d_{1}^{\prime}$ and $d_{2}^{\prime}$ show that the Seifert surface $D_{1}^{1} \cup D_{2}^{1}$ is of type I. Therefore $K$ is a 2-bridge knot $K(4 \alpha \beta-1,2 \alpha)$, and the Seifert surface of type II is isotopic to that of type I. Next suppose $\ell_{1}=\ell_{2}$ is a torus knot of type $(1, \alpha)$ on $\partial V_{2}$. The same arguments as above show, after interchanging the roles of $V_{1}$ and $V_{2}$, that $K$ is a 2-bridge knot $K(4 \alpha \beta-1,2 \alpha)$, and the Seifert surface of type II is isotopic to that of type I. Next suppose $\ell_{1}=\ell_{2}$ is a torus knot of type $(p, q)$ on $\partial V_{2}$, where $p \neq 1$ and $q \neq 1$. Let $R_{0}$ be the annulus $H \cap\left(S^{3}-\right.$ int $\left.N\left(\ell_{1}\right)\right)$. The annuli $D_{1}^{1}, D_{2}^{1}$ and the torus $H$ separate the torus $\partial N\left(\ell_{1}\right)$ into four annuli $R_{1}, R_{2}, R_{3}$ and $R_{4}$. Assume $R_{1} \cap d_{1}^{\prime} \neq \emptyset$ and $R_{2} \cap d_{2} \neq \emptyset$. Let $R^{\prime}$ be the union of the surfaces $\left(D_{1}^{1} \cup D_{2}^{1}\right) \cap\left(S^{3}-i n t N\left(\ell_{1}\right)\right), R_{0}, R_{1}$ and $R_{2}$. Let $U$ be the solid torus which is the union of $N\left(R^{\prime}\right), N\left(d_{1}^{\prime}\right)$ and $N\left(d_{2}\right)$. The same arguments as in the previous paragraph show that $K$ is a tunnel number one knot $K(8 \beta, 4 \beta+1 ; p, q)$. The union of the $\operatorname{arcs} \partial d_{1}^{\prime} \cap D_{1}^{1}, \partial d_{2} \cap D_{2}^{1}$ and the loop $\ell_{1}$ is a deformation retract of $D_{1}^{1} \cup D_{2}^{1}$. Let $\tau$ be the arc which is a union of the arcs $\partial d_{1}^{\prime} \cap D_{1}^{1}$ and $\partial d_{2} \cap D_{2}^{1}$. The arc $\tau$ may be regarded as an unknotting tunnel for the torus knot $\ell_{1}$, so $S^{3}-\operatorname{int} N\left(D_{1}^{1} \cup D_{2}^{1}\right)$ is a genus two handlebody. Hence the Seifert surface of type II is not isotopic to that of type I for $K(8 \beta, 4 \beta+1 ; p, q)$. 


\section{ACKNOWLEDGEMENTS}

The author would like to express his gratitude to Professor Cameron Gordon, Professor Masakazu Teragaito, Professor Hiroshi Goda and Professor Chuichiro Hayashi for their helpful comments.

\section{ADDED IN PROOF}

After the author had completed this work, Martin Scharlemann proved Conjecture 1.2 by using Theorem 1.1 in his preprint "There are no unexpected tunnel number one knots of genus one".

\section{REFERENCES}

1. G. Burde, H. Zieschang, Neuwirthsche Knoten und Flächenabbildungen, Abh. Math. Sem. Univ. Hamburg 31 (1967) 239-246. MR 37:4803

2. G. Burde, H. Zieschang, Knots, de Gruyter Studies in Mathematics 5, Walter de Gruyter \& Co., Berlin-New York (1985). MR 87b:57004

3. H. Doll, A generalized bridge number for links in 3-manifolds, Math. Ann. 294 (1992), 701717. MR 93i:57023

4. M. Eudave-Muñoz, On nonsimple 3-manifolds and 2-handle addition, Topology Appl. 55 (1994) 131-152. MR 95e:57029

5. H. Goda, M. Teragaito, Tunnel number one genus one non-simple knots, Tokyo J. Math. 22 (1999) 99-103. MR 2000j:57011

6. C. Hayashi, Genus one 1-bridge positions for the trivial knot and cabled knots, Math. Proc. Cambridge Philos. Soc. 125 (1999) 53-65. MR 99j:57005

7. K. Morimoto, M. Sakuma, On unknotting tunnels for knots, Math. Ann. 289 (1991) 143-167. MR 92e:57015

8. K. Morimoto, M. Sakuma, Y. Yokota, Examples of tunnel number one knots which have the property " $1+1=3$ ", Math. Proc. Cambridge Philos. Soc. 119 (1996) 113-118. MR 96i:57007

9. K. Murasugi, Knot theory and its applications, Birkhäuser Boston, Inc, Boston, MA (1996). MR 97g:57011

10. M. Ozawa, Satellite knots of free genus one, J. Knot Theory Ramifications 8 (1999) 27-31. MR 2000b:57012

11. W. Whitten, Isotopy types of knot spanning surfaces, Topology 12 (1973) 373-380. MR 51:9046

Graduate School of Mathematical Sciences, University of Tokyo, Tokyo 153-8914, JAPAN

E-mail address: matsuda@ms.u-tokyo.ac.jp 